\title{
Multi-Objective Optimization Dispatch Based Energy Management of A Microgrid Running Under Grid Connected and Standalone Operation Mode
}

\author{
Marouane Lagouir*, Abdelmajid Badri, Yassine Sayouti \\ Department of Electrical Engineering, EEA\&TI Laboratory, Faculty of Science and Technology (FSTM), Hassan II University of \\ Casablanca, BP 146 Mohammedia 20650, Morocco
}

\begin{abstract}
This paper presents a novel optimization approach for a day-ahead power management and control of a DC microgrid (MG). The multi-objective optimization dispatch (MOOD) problem involves minimizing the overall operating cost, pollutant emission levels of $\left(\mathrm{NO}_{\mathrm{x}}, \mathrm{SO}_{2}\right.$ and $\left.\mathrm{CO}_{2}\right)$ and the power loss cost of the conversion devices. The weighted sum method is selected to convert the multi-objective optimization problem into a single optimization problem. Then, analytic hierarchy process (AHP) method is applied to determine the weight coefficients, according to the preference of each objective function. The system's performance is evaluated under both grid connected and standalone operation mode, considering power balancing, high level penetration of renewable energy, optimal scheduling of charging/discharging of battery storage system, control of load curtailment and the system technical constraints. Ant lion optimizer (ALO) method is considered for handling MOOD, and the performance of the proposed algorithm is compared with other known heuristic optimization techniques. The simulation results prove the effectiveness and the capability of the developed approach to deal better with the coordinated control and optimization dispatch problem. They also revealed that economically running the MG system under grid connected mode can reduce the overall cost by around $4.70 \%$ compared to when it is in standalone operation mode.
\end{abstract}

Keywords: Microgrid, multi-objective optimization dispatch, operating cost, pollutant emission levels, power loss cost, weighted sum method, analytic hierarchy process, renewable energy, ant lion optimizer, heuristic optimization techniques.

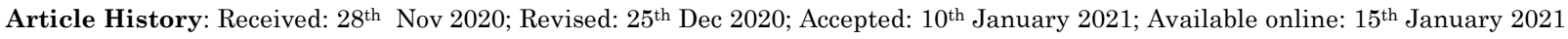
How to Cite This Article: Lagouir, M., Badri, A. and Sayouti, Y. (2021) Multi-Objective Optimization Dispatch Based Energy Management of A Microgrid Running Under Grid Connected and Standalone Operation Mode. Int. Journal of Renewable Energy Development, 10(2), 333-343. https://doi.org/10.14710/ijred.2021.34656

\section{Introduction}

Nowadays, with the increasing demand for high quality, efficient and secure electric power system, the adoption of microgrid (MG) concept has received considerable attention. A MG can be described as an integrated energy system consisting of interconnected loads and distributed energy resources (DERs). Generally, a microgrid can operate either connected to the main grid through selling or purchasing power (Parisio et al. 2014), or in standalone operating mode in case of emergencies or remote areas. A typical MG is generally composed by: conventional energy sources (CESs), renewable energy sources (RESs), energy storage systems, conversion electronic devices, monitoring systems and electrical loads.

The coordinated operation of a MG is a challenging task, therefore an energy management system (EMS) plays a vital role to improve power supply quality, reliability, and secure decisions for the MG operations. The purpose of an energy management in MGs is to find the optimal unit commitment (UC) and power dispatch of the available generating units, to achieve selected objectives. In standalone MG operation's mode, the main purpose is to meet the system load demand, at minimum operating cost, whilst under grid-connected mode a commonly adopted objective is the maximization of MG's economic benefits (Olivares et al. 2011).

Several studies have been developed to solve the coordinated control and optimization dispatch problems in MGs. In (Kyriakarakos et al. 2012; Vivas et al. 2020; Garcia et al. 2013; Lagouir et al. 2019) a fuzzy logic control system has been proposed for optimal energy management of a MG. The fuzzy logic approach is suitable for complex nonlinear system and it is a promising solution for its robustness and good real-time performance. Nemati et al. (2018) propose two improved methods to schedule both the unit commitment and economic dispatch of MGs. The functionality of the developed real-coded genetic algorithm (GA) and the enhanced mixed integer linear programming (MILP) based method have been evaluated and compared under both grid connected and standalone mode, considering operation cost optimization, minimum emissions level and combined mode. The obtained results demonstrate better performance of GA compared to MILP. Aghajani et al. (2018) proposed a multi objective particle swarm optimization method (MOPSO) for day-ahead energy management, considering operation cost and

\footnotetext{
* Corresponding author: lagouir.mar1@gmail.com
} 
emission rate as objective functions, then the results are compared with non-dominated sorting genetic algorithm II (NSGA-II) technique to show the superior performance of the proposed method. In (Nwulu et al. 2015), the authors discuss the implementation of a novel control approach to optimize two objective functions, considering the integration of a game theory based demand response (DR) program into the dynamic economic emission dispatch (DEED) problem. The obtained results indicate the practical benefits of the developed model compared with independent optimization of DR or DEED.

Many intelligent optimization techniques are used to handle the multi-objective optimization dispatch (MOOD). Fireworks algorithm with gravitational search operator (MFAGSO) (Wang et al. 2017), MOPSO (Aghajani et al. 2018) method, ant lion optimizer (ALO) algorithm (Hatata et al. 2018), NSGA-II optimization method (Contreras et al. 2019), strength pareto evolutionary algorithm (SPEA) (Yuan et al. 2017) and so on.

The purpose of this work is the development and implementation of a two-level EMS for optimal power management and control of a MG. Each layer with a specific function and they collaborate to ensure more flexible energy scheduling and control. Three conflicting objective functions are considered to be minimized, which are: the overall operating cost, the pollutant gas emissions cost of $\left(\mathrm{NO}_{\mathrm{x}}, \mathrm{SO}_{2}, \mathrm{CO}_{2}\right)$, and the power loss cost of the conversion devices, taking into account the technical constraints of the system (Taha et al. 2018). Ant lion optimizer algorithm is selected to solve the MOOD problem, then the achieved results are compared with other well know heuristic algorithms include artificial bee colony (ABC) (Karaboga et al. 2009), novel bat algorithm (NBA) (Meng et al. 2015) and particle swarm optimization (PSO) technique to verify the robustness and reliability of the proposed approach.

The organization of the paper is as follows. The global system overview is shown in Section 2. Section 3 details the formulation of the optimization problem. Section 4 describes the proposed energy management approach. In Section 5, the simulation results are presented and discussed. Finally, the conclusion is reported in Section 6.

\section{Global system overview}

Fig. 1 shows the overall architecture of the studied MG. It can be described as a cluster of various distributed energy resources, such as renewable and conventional energy sources, energy storage system (ESS) (Jiang et al. 2013), power conversion devices and variable demands (VDs) which is perceived by the main grid as a single element (Olivares et al. 2011). The interconnection between the main grid and the studied MG system is ensured through a point of common coupling (PCC). Finally, all those units are connected to a centralized EMS developed for coordinated operation and optimization dispatch of the MG system. The renewable energy sources combine photovoltaic arrays (PV) and wind turbine (WT), while conventional energy sources are consisting of a diesel generator (DG), a fuel cell (FC), and a microturbine (MT). Considering the stochastic nature of RESs, a battery system is served as a backup to maintain power balance and to improve the reliability of the MG (Garcia et al. 2014). The mathematical modelling of the consisting components is detailed in the following subsections.

\subsection{Power generation resources}

\subsubsection{Solar energy system}

The output power of PV generating unit is expressed as a function of incident irradiance $G$ and cell temperature $T$ using the following formula (Taha et al. 2018; Moradi et al. 2018; Wang et al. 2017):

$P_{P V}=F_{P V} \times P_{P V, R} \times \frac{G}{G_{S T C}}\left[1+\alpha_{T}\left(T-T_{S T C}\right)\right]$

where:

- $\quad F_{P V}$ is the factor reflecting shading, and wiring losses,

- $\quad \alpha_{T}$ represents the temperature coefficient, and

- $G_{S T C}$ and $T_{S T C}$ represent the incident irradiance and the PV cell temperature under Standard Test Conditions, respectively.

\subsubsection{Wind energy system}

The output power of the WT unit can be approximated as a function of the wind speed $V$ and the rated output power $P_{W T, R}$ given as (Moradi et al. 2018; Shen et al. 2016; Kiptoo et al. 2020):

$$
\left\{\begin{array}{c}
P_{W T}=0 ; V<V_{C I} \text { or } V>V_{C O} \\
P_{W T}=P_{W T, R}\left(\frac{V^{3}-V_{C I}^{3}}{V_{R}^{3}-V_{C I}^{3}}\right) ; V_{C I}<V<V_{R} \\
P_{W T}=P_{W T, R} ; V_{R}<V<V_{C O}
\end{array}\right.
$$

where:

- $P_{W T}$ is the actual output power, and

- $V_{R}, V_{C I}, V_{C O}$ represent the rated, cut-in and cutout wind speed, respectively.

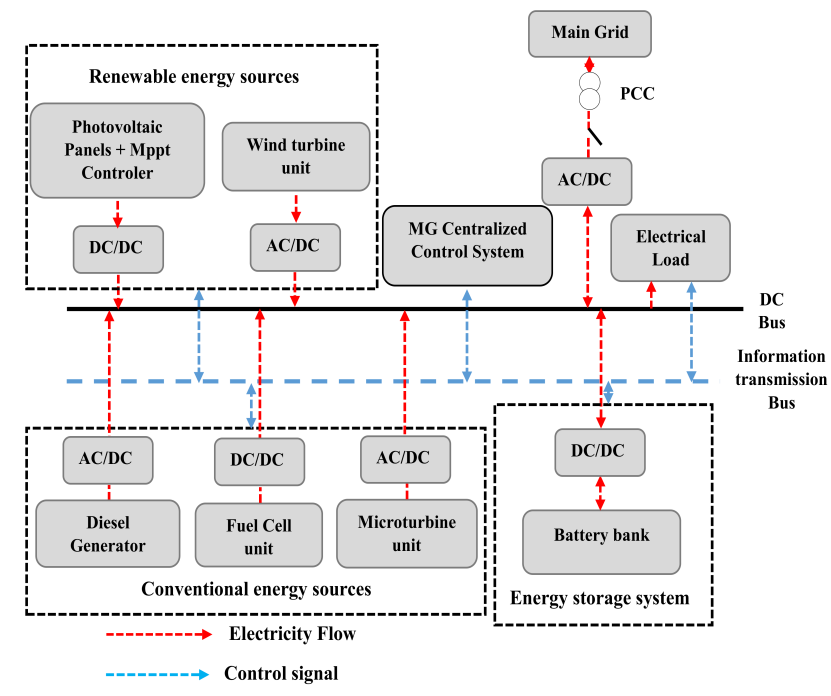

Fig. 1 Studied microgrid architecture 


\subsection{Energy Storage System}

In the studied MG system, the battery is employed as a backup to store the excess of power when the supply from RESs exceeds load demand. On the other hand, the battery will provide energy to the system when the supply from RESs cannot meet the required demand. The State of charge (SoC) for battery is considered as an important parameter to be controlled. The variation of the battery SoC is expressed by eq. (3) given as (AL-Sakkaf et al. 2019):

$S o C_{t}=S o C_{0}+\frac{1}{Q} \int_{t_{0}}^{t_{f}} i d t$

where:

- $\int i d t$ is the battery bank instantaneous capacity in $\mathrm{Ah}$,

- $\quad$ is the battery bank maximal capacity in Ah.

To maintain a longer life-time of the battery, monitoring its $\mathrm{SoC}$ is essential to prevent it from any overcharging or under discharging. The associated inequality constraint is given as follows (Moradi et al. 2018; Jin et al. 2017):

$$
\mathrm{SoC}_{\mathrm{Min}} \leq \mathrm{SoC}_{t} \leq \mathrm{SoC}_{\mathrm{Max}}
$$

The output power of the battery storage at time $t$ should be maintained within the minimum and maximum limits as follows (Reddy et al. 2015):

$P_{E S S_{\min }} \leq P_{E S S}(t) \leq P_{E S S_{\max }}$

Where a positive value of $P_{E S S}$ corresponds to a battery in discharging mode. Whilst the battery is in charging mode when $P_{E S S}$ is negative (Reddy et al. 2017). Finally, the generation of the battery is equal to zero whenever the battery SoC is less than $20 \%$, or in case when it's SoC $\geq$ $80 \%$ and the supply of both RESs is sufficient to meet the required demand.

\section{Formulation of the optimization problem}

\subsection{Objective functions}

In the studied MG, the three contradictory objective functions of optimization dispatch to be minimized are: the operating cost of MG system. The second level objective function is the pollutant gas emissions cost of nitrogen oxide, sulfur dioxide and carbon dioxide $\left(\mathrm{NO}_{\mathrm{x}}, \mathrm{SO}_{2}\right.$ and $\mathrm{CO}_{2}$ ) respectively. Finally, the power loss cost of the conversion devices is defined as the third level objective function.

The analytic hierarchy process (AHP) is used to formulate judgment matrix and determine the weight coefficients, that converting the multi-objective functions into a single objective function (Liu et al. 2015). The mathematical model of the objective function is formulated by eq. (6) given as (Liu et al. 2015):

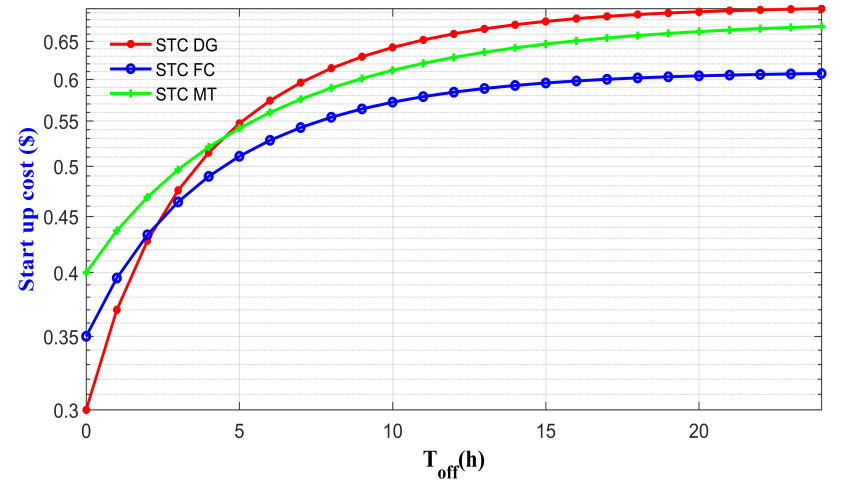

Fig. 2 Start-up cost of the dispatchable sources as a function of the time the unit has been off

$\min O F_{M G}=\omega_{1} C_{1}+\omega_{2} C_{2}+\omega_{3} C_{3}$

\subsubsection{Operating cost function}

The operating cost of the studied MG system, is formulated as the sum of the fuel cost of generators, startup cost, maintenance cost, and exchanged power cost with the main grid, given as (Nemati et al. 2018; Moradi et al. 2018; Wang et al. 2017; Alvarado-Barrios et al. 2019; Mohamed et al 2012):

$$
\begin{aligned}
C_{1} & =\sum_{i=1}^{N}\left(C F_{i}\left(P_{i}\right)+O M_{i}\left(P_{i}\right)+S T C_{i}\right) \\
& +\sum_{i=1}^{N}\left(C P E_{i}-R S E_{i}\right)
\end{aligned}
$$

\section{a) Fuel cost of generating units}

The fuel cost consumed by each conventional energy source is approximated to be a quadratic function of the active power from the generating unit $i$ (Nemati et al. 2018; Moradi et al. 2018; Wang et al. 2017; Jin et al. 2017; Alvarado-Barrios et al. 2019):

$C F_{i}\left(P_{i}(t)\right)=\alpha_{i}+\beta_{i} P_{i}(t)+\gamma_{i} P_{i}^{2}(t)$

where:

- $\alpha_{i}, \beta_{i}, \gamma_{i}$ are cost coefficients of generator $i$,

- $P_{i}$ is the actual output power, and

- $\quad C F_{i}$ is the fuel cost of the generating unit $i$ (DG or FC or MT),

\section{b) Operation and maintenance cost}

The O\&M cost of each conventional energy source is expressed as a linear function of the actual output power $P_{i}$ given as (Moradi et al. 2018; Mohamed et al. 2012):

$O M_{i}=\sum_{i=1}^{N} K_{O M_{i}} P_{i}$

where:

- $K_{O M_{i}}$ is the cost coefficient for each generating unit $i$. 


\section{c) Start-up cost}

The start-up cost of each dispatchable unit $i$ can be calculated as a function of the time the generator has been off (Moradi et al. 2018; Wang et al. 2017; Alvarado-Barrios et al. 2019), as specified in eq. (10). Fig. 2 represents the start-up cost of DG, FC and MT, respectively.

$S T C_{i}=\sigma_{i}+\delta_{i}\left[1-\exp \left(-\frac{T_{o f f, i}}{\tau_{i}}\right)\right]$

where:

- $\sigma_{i}$ is hot start-up cost,

- $\delta_{i}$ is cold start-up cost,

- $\tau_{i}$ is the constant of unit cooling time, and

- $T_{\text {off }, i}$ is unit shutdown duration.

\section{d) Exchanged power cost with the main grid}

In grid connected mode, the MG may exchange power with the main grid. Therefore, when the load demand exceeds the combination supply limitation of RESs and CESs, there is a cost to be paid for the purchased power. On the other hand, there is income from the surplus power sold to the main grid. To model the purchased and sold power eq. (11) are considered (Mohamed et al 2012). The electricity price profile within a day is shown in Fig. 3.

$C P E_{i}=C_{p} \times \max \left(P_{D}-P_{i}, 0\right)$

$R S E_{i}=C_{s} \times \max \left(P_{i}-P_{D}, 0\right)$

where:

- $C_{p}$ is the price of the purchased power in $[\$ / \mathrm{kwh}]$,

- $C_{s}$ is the price of the sold power in [\$/kwh],

- $P_{D}$ represents the load demand,

- $\quad C P E$ is cost of purchasing electricity in $[\$ / \mathrm{h}]$, and

- $R S E$ is revenue of selling electricity in $[\$ / \mathrm{h}]$.

\subsubsection{Pollutant gas emissions cost function}

The second term of eq. (6) represents the pollutant gas emissions cost of $\mathrm{NO}_{\mathrm{x}}, \mathrm{SO}_{2}$ and $\mathrm{CO}_{2}$, respectively. It is expressed as a linear function of produced power (Liu et al. 2015; Wu et al. 2014):

$C_{2}=\sum_{i=1}^{N} \sum_{k=1}^{M} \chi_{k}\left(E F_{i k} P_{i}\right)+\sum_{k=1}^{M} \chi_{k}\left(E F_{\text {Gridk }} P_{\text {Grid }}\right)$

where:

- $E F_{\text {Gridk }}, E F_{i k}$ are emission factors of grid and generating unit $i$ for emission type $k$ in $[\mathrm{kg} / \mathrm{kwh}]$,

- $\chi_{k}$ is externality costs of emission type $k$ in $[\$ / \mathrm{kg}]$,

- $\quad N$ number of generating units,

- $\quad M$ is emission types $\left(\mathrm{NO}_{\mathrm{x}}\right.$ or $\mathrm{SO}_{2}$ or $\left.\mathrm{CO}_{2}\right)$, and

- $\quad P_{i}, P_{\text {Grid }}$ are the produced power from generator $i$ and main grid in $[\mathrm{kW}]$.

The operating cost parameters and the externality costs, emission factors of both CESs and the main grid used in this study are provided in Table 1,2. As specified in (Nemati et al. 2018; Mohamed et al 2012, Moradi et al. 2018 Alvarado-Barrios et al. 2019).

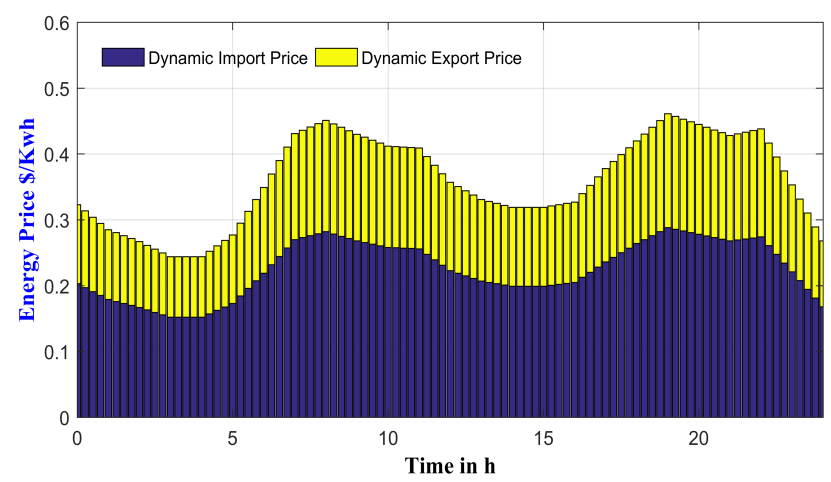

Fig. 3 Daily electricity price profile

Table 1

Conventional energy sources operating cost parameters

\begin{tabular}{ccccccccc} 
& \multicolumn{2}{c}{ P rated } & \multicolumn{2}{c}{ Fuel or gas cost coefficients } & \multicolumn{3}{c}{ Start-up cost } & \multicolumn{2}{c}{ O\&M cost } \\
\cline { 2 - 9 } & $P_{i}[\mathrm{Kw}]$ & $\alpha_{i}[\$ / \mathrm{h}]$ & $\beta_{i}[\$ / \mathrm{kwh}]$ & $\gamma_{i}\left[\$ / \mathrm{kw}^{2} \mathrm{~h}\right]$ & $\sigma_{i}[\$ / \mathrm{h}]$ & $\delta_{i}[\$ / \mathrm{h}]$ & $\tau_{i}$ & $K_{O M_{i}}[\$ / \mathrm{kwh}]$ \\
\hline DG & 40 & 2.22 & 0.2328 & 0.0024 & 0.3 & 0.4 & 5.2 & 0.01258 \\
FC & 50 & 0.1037 & 0.1855 & 0.0009 & 0.35 & 0.26 & 5.2 & 0.00419 \\
MT & 65 & 2.898 & 0.2668 & - & 0.4 & 0.28 & 7.1 & 0.00587 \\
\hline
\end{tabular}

Table 2

Externality costs and emission factors of conventional energy sources and the main grid

\begin{tabular}{cccccc}
\hline Emission type & External Costs $[\$ / \mathbf{k g}]$ & $\mathbf{D G}[\mathbf{k g} / \mathbf{M W h}]$ & $\mathbf{F C}[\mathbf{k g} / \mathbf{M W h}]$ & MT $[\mathbf{k g} / \mathbf{M W h}]$ & Main Grid [kg/MWh] \\
\hline $\mathrm{NO}_{\mathbf{x}}$ & 9.1714 & 9.8883 & 0.0136 & 0.1995 & 1.6021 \\
$\mathrm{SO}_{2}$ & 2.1617 & 0.2059 & 0.0027 & 0.0036 & 1.8016 \\
$\mathrm{CO}_{2}$ & 0.0305 & 0.6495 & 0.4889 & 0.7239 & 0.8891 \\
\hline
\end{tabular}




\subsubsection{Power loss cost function}

The power loss cost of the conversion devices is considered as the third level of the MOOD problem. The mathematical model of the power loss cost function is expressed as follows (Wu et al. 2019):

$$
\begin{aligned}
C_{3} & =\sum_{i=1}^{N} C_{\text {loss }} P_{\text {loss }} \\
& =C_{\text {loss }} \sigma_{\text {loss }}\left(P_{R E S_{s}}(t)+P_{E S S}(t)+P_{C E S_{s}}(t)+P_{\text {Grid }}(t)\right)
\end{aligned}
$$

where:

- $C_{\text {loss }}$ is the penalty of the power loss cost function in $[\$ / \mathrm{kwh}]$,

- $\sigma_{\text {loss }}$ is the coefficient of conversion power loss (assumed to be $2 \%$ ), and

- $P_{\text {loss }}$ is power loss of conversion device related to the generating unit $i$.

\subsection{Analytic hierarchy process for weights calculation}

The linear additivity of the objective functions is not applicable to solve the MOOD problem (Wu et al. 2016). Therefore, a weighting approach based on weighted sum method (Augusto et al. 2012) is considered to transform the multi-objective optimization problem into a singleobjective problem. The analytic hierarchy process is a subjective technique used to determine the weight coefficients, according to a priori preference of each objective function.

The first step in the AHP method involves the formulation of judgement matrix $J$ according to the importance of each objective. It is given as follows:

$J=\left(\begin{array}{ccc}1 & 3 & 5 \\ 1 / 3 & 1 & 3 \\ 1 / 5 & 1 / 3 & 1\end{array}\right)$

After matrix processing using AHP (Triantaphyllou et al. 1995), the obtained weight coefficients are $\omega_{1}=0.6370$,

$\omega_{2}=0.2583, \omega_{3}=0.1047$ (Liu et al. 2015).

The following constraint is to be taken into account:

$\omega_{i} \geq 0$ and $\sum_{i=1}^{3} \omega_{i}=1$

To verify the consistency of the obtained weight coefficients, the following parameters are calculated:

$$
\lambda_{\max }=3.01851, C I=0.01925, C R=0.03702<0.1
$$

where:

- $\lambda_{\max }$ represents the maximum eigenvalue,

- $\quad C I$ is the consistency index, and

- $\quad C R$ is the consistency ratio.

According to (Triantaphyllou et al. 1995), obtained a consistency ratio less than $10 \%$, valid the consistency of the calculated weights coefficients and therefore of the formulated judgment matrix.

\subsection{System constraints}

The operation of the system's components is subject to several constraints.

To maintain power balance, the total generation of all MG's components, including the exchanged power with the main grid and the ESS should be equal to the actual load demand plus power loss. This equality is given by:

$$
P_{D}(t)+\mathrm{P}_{\text {loss }}(t)=P_{\text {Grid }}(t)+P_{C E S_{s}}(t)+P_{E S S}(t)+P_{R E S_{s}}(t)
$$

The power supply limitation of the consisting generation units is bounded according to the following constraints:

$$
\begin{gathered}
P_{D G_{M i n}} \leq P_{D G} \leq P_{D G_{M a x}} \\
P_{F C_{M i n}} \leq P_{F C} \leq P_{F C_{M a x}} \\
P_{M T_{M i n}} \leq P_{M T} \leq P_{M T_{M a x}} \\
P_{G r i d_{M i n}} \leq P_{G r i d} \leq P_{G r i d_{M a x}} \\
0 \leq P_{P V} \leq P_{P V_{M p p t}} \\
0 \leq P_{W T} \leq P_{W T_{M p p t}}
\end{gathered}
$$

\subsection{Load demand model}

In the proposed MG architecture, two kinds of demands are considered, critical demand, and controllable demand, where it has flexible magnitude.

For the controllable demand, it is assumed that, the demand reduction (DR) is limited to the magnitude $M(t)$, and thus, DR maximum magnitude limits can be expressed as:

$0 \leq D R(t) \leq M(t)$

\section{Energy management system}

The main objective of this research work, is the development of a novel energy management system, for real time power management optimization and control of MG. Fig. 4 illustrates the proposed EMS. It is designed to operate in a two-level control structure. The upper layer comprises a fuzzy logic system (FLS) implemented in order to define the participation of each RESs, optimal scheduling of charging/discharging of ESS (Murty et al. 2020), the decision to switch on or off the CESs, load curtailment control and the interactive mode between the grid and the MG. In this strategy the CESs are dispatched only when the load demand exceeds the power supply limitation of both RESs and ESS. In each step, the fuzzy scheduling system acquires current SoC data, RESs power generation, price of electricity and the load demand. For each combination of inputs, the FLS decides to activate or not the CESs. The FLS has four inputs; battery SoC, operating state of the battery (charging or discharging mode), normalized electricity prices (NEP) and normalized 
net power (NNP) that is defined as the difference between actual power demand and RESs power generations, and has two output which represents the operating mode of each DERs and the optimal charging/discharging power of ESS, respectively.

The abbreviations L, M, H and $\mathrm{VH}$ are used to describe membership functions low, medium, high and very high, of the normalized electricity price's input respectively. The terms $\mathrm{HC} / \mathrm{D}, \mathrm{MC} / \mathrm{D}$ and $\mathrm{LC} / \mathrm{D}$, in output membership function stand for high, medium and low charging or discharging of ESS. Finally, the abbreviations NE, ZO and $\mathrm{PO}$, respectively correspond to negative, zero and positive normalized net power. Fig. 5 presents the structure of the control system based fuzzy logic. Finally, the detailed fuzzy scheduling rules for ESS are shown in Table 3.

As regards the bottom layer is implemented to solve the multi-objective optimization dispatch problem, considering minimization the operating cost, the pollutant gas emissions cost and the power loss cost as objective functions. Ant lion optimizer algorithm is selected for handling the MOOD issue. The obtained simulation results are compared with those provided by other heuristic methods such as PSO, ABC and NBA algorithm.

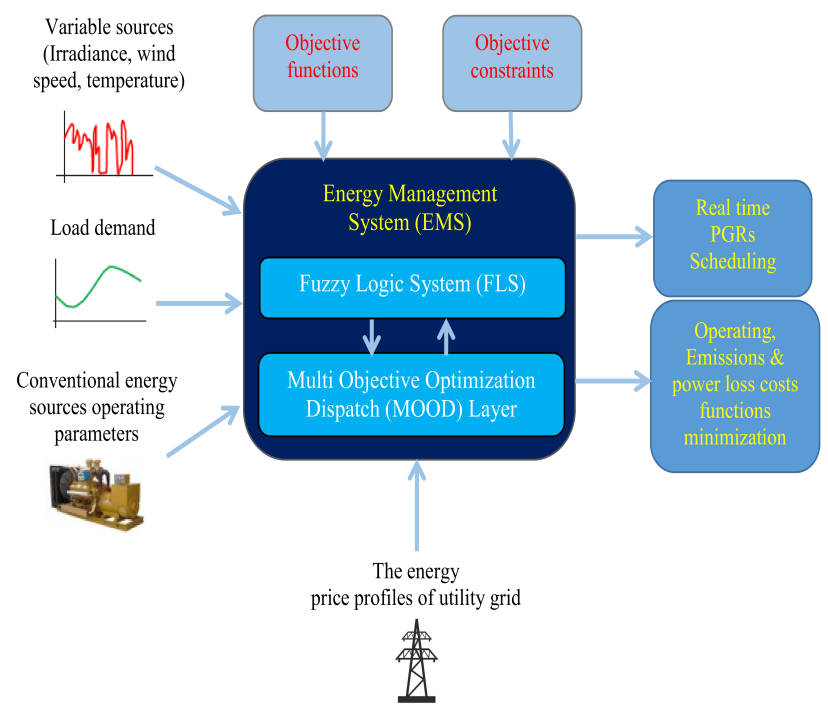

Fig. 4 Overall energy management system

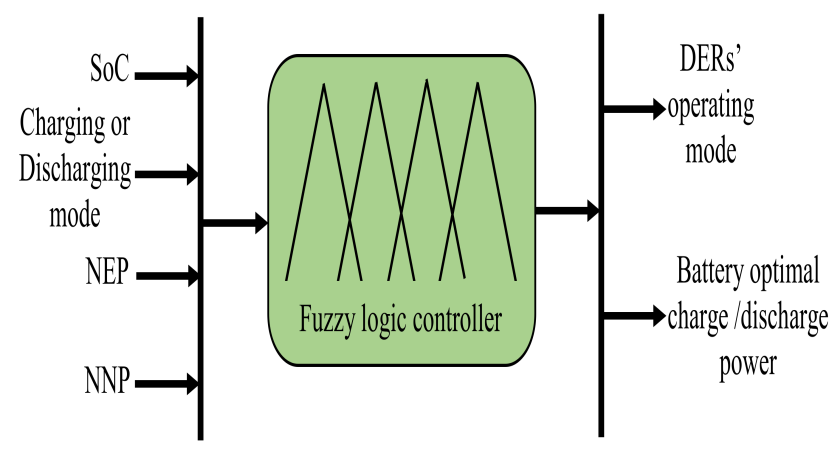

Fig. 5 Structure of fuzzy logic control system

\subsection{Ant lion optimizer algorithm}

Ant lion optimizer is originally attributed to Mirjalili, it is a novel developed population based search algorithm, it mimics the hunting process of antlion in nature (Mirjalili 2015). The main steps considered to model the hunting behavior of antlion are detailed in the following subsections (Hatata et al. 2018; Alazemi et al. 2019):

\subsubsection{Random walks of ants}

Considering that ants move randomly when looking for food. The mathematical modelling of the movement behavior is given as (Hatata et al. 2018; Mirjalili 2015; Alazemi et al. 2019; Kamboj et al. 2017):

$$
X(t)=\left[\begin{array}{l}
0, \text { cumusum }\left(2 r\left(t_{1}\right)-1\right), \\
\operatorname{cumusum}\left(2 r\left(t_{2}\right)-1\right), \\
\ldots, \text { cumusum }\left(2 r\left(t_{\text {Iter }_{\text {Max }}}\right)-1\right)
\end{array}\right]
$$

where:

- $X(t)$ represents the random walks of ants,

- Iter $_{\text {Max }}$ is the maximum number of iteration, and

- $t$ refers to the step of random walk.

$r(t)$ is a stochastic function defined as follows:

$r(t)=\left\{\begin{array}{l}1 \text { if } \text { rand }>0.5 \\ 0 \text { otherwise }\end{array}\right.$

where: rand is a random number uniformly distributed in $[0,1]$.

To guarantee that the random walks of ants are inside the search space, they are normalized using min-max normalization method given as (Kamboj et al. 2017):

$X_{i}^{t}=\frac{\left(X_{i}^{t}-a_{i}\right)\left(d_{i}-c_{i}^{t}\right)}{\left(d_{i}^{t}-a_{i}\right)}+c_{i}$

where:

- $a_{i}$ and $d_{i}$ indicate the minimum and the maximum of random walk of $i^{\text {th }}$ variable, respectively, and

- $\quad c_{i}^{t}$ and $d_{i}^{t}$ are the minimum and the maximum of $i^{\text {th }}$ variable at $t^{\text {th }}$ iteration, respectively.

\subsubsection{Building antlion trap}

To model the antlion's hunting capability, roulette wheel is employed (Kamboj et al.2017). This operator is used by the ALO algorithm for selection of antlions based on their fitness. According to (Mirjalili 2015), the roulette wheel mechanism results more chance that antlions catch preys. 
Table 3

Fuzzy rules based ESS optimal scheduling

\begin{tabular}{|c|c|c|c|c|c|c|c|c|c|c|c|c|c|c|c|c|c|c|c|c|c|c|c|c|}
\hline \multirow{3}{*}{$\frac{\text { SoC }}{\text { NEP }}$} & \multicolumn{8}{|c|}{ NE } & \multicolumn{8}{|c|}{$\mathbf{Z O}$} & \multicolumn{8}{|c|}{ PO } \\
\hline & \multicolumn{4}{|c|}{ Charging Mode } & \multicolumn{4}{|c|}{$\begin{array}{c}\text { Discharging } \\
\text { Mode }\end{array}$} & \multicolumn{4}{|c|}{ Charging Mode } & \multicolumn{4}{|c|}{$\begin{array}{c}\text { Discharging } \\
\text { Mode }\end{array}$} & \multicolumn{4}{|c|}{ Charging Mode } & \multicolumn{4}{|c|}{$\begin{array}{l}\text { Discharging } \\
\text { Mode }\end{array}$} \\
\hline & $\mathrm{L}$ & $\mathrm{M}$ & $\mathrm{H}$ & $\mathrm{VH}$ & $\mathrm{L}$ & $\mathrm{M}$ & $\mathrm{H}$ & VH & $\mathrm{L}$ & M & $\mathrm{H}$ & VH & $\mathrm{L}$ & $\mathrm{M}$ & $\mathrm{H}$ & $\mathrm{VH}$ & $\mathrm{L}$ & M & $\mathrm{H}$ & $\mathrm{VH}$ & $\mathrm{L}$ & $\mathrm{M}$ & $\mathrm{H}$ & $\mathrm{VH}$ \\
\hline Low SoC & $\mathrm{HC}$ & $\mathrm{HC}$ & $\mathrm{HC}$ & $\mathrm{HC}$ & $\mathrm{ZO}$ & $\mathrm{ZO}$ & $\mathrm{ZO}$ & $\mathrm{ZO}$ & $\mathrm{HC}$ & $\mathrm{HC}$ & $\mathrm{MC}$ & $\mathrm{MC}$ & $\mathrm{ZO}$ & $\mathrm{ZO}$ & $\mathrm{ZO}$ & $\mathrm{ZO}$ & $\mathrm{HC}$ & $\mathrm{MC}$ & $\mathrm{LC}$ & $\mathrm{LC}$ & $\mathrm{ZO}$ & $\mathrm{ZO}$ & $\mathrm{ZO}$ & $\mathrm{ZO}$ \\
\hline Medium Low & $\mathrm{HC}$ & $\mathrm{HC}$ & $\mathrm{MC}$ & $\mathrm{MC}$ & $\mathrm{ZO}$ & $\mathrm{ZO}$ & $\mathrm{LD}$ & $\mathrm{LD}$ & $\mathrm{HC}$ & $\mathrm{MC}$ & $\mathrm{LC}$ & $\mathrm{LC}$ & $\mathrm{ZO}$ & $\mathrm{ZO}$ & $\mathrm{LD}$ & $\mathrm{MD}$ & $\mathrm{MC}$ & MC & $\mathrm{LC}$ & $\mathrm{LC}$ & $\mathrm{LD}$ & $\mathrm{LD}$ & MD & HD \\
\hline $\begin{array}{l}\text { Medium } \\
\text { High }\end{array}$ & $\mathrm{LC}$ & $\mathrm{LC}$ & $\mathrm{ZO}$ & $\mathrm{ZO}$ & $\mathrm{ZO}$ & $\mathrm{ZO}$ & $\mathrm{LD}$ & $\mathrm{MD}$ & $\mathrm{LC}$ & $\mathrm{ZO}$ & $\mathrm{ZO}$ & $\mathrm{ZO}$ & $\mathrm{ZO}$ & $\mathrm{LD}$ & $\mathrm{MD}$ & HD & $\mathrm{LC}$ & $\mathrm{ZO}$ & $\mathrm{ZO}$ & $\mathrm{ZO}$ & $\mathrm{MD}$ & $\mathrm{MD}$ & HD & HD \\
\hline High & $\mathrm{ZO}$ & $\mathrm{ZO}$ & $\mathrm{ZO}$ & $\mathrm{ZO}$ & LD & $\mathrm{LD}$ & MD & $\mathrm{MD}$ & $\mathrm{ZO}$ & $\mathrm{ZO}$ & $\mathrm{ZO}$ & $\mathrm{ZO}$ & MD & MD & HD & HD & $\mathrm{ZO}$ & $\mathrm{ZO}$ & $\mathrm{ZO}$ & $\mathrm{ZO}$ & MD & HD & HD & HD \\
\hline
\end{tabular}

\subsubsection{Trapping in pits}

Assuming that, the random movement of ants is affected by antlions' traps, trapping the ant in antlion's pit is modelled using the following equations (Mirjalili 2015):

$c_{i}^{t}=$ Antlion $_{j}^{t}+c^{t}$

$d_{i}^{t}=$ Antlion $_{j}^{t}+d^{t}$

where:

- $\quad c^{t}$ and $d^{t}$ are the minimum and the maximum of all variables at the $t^{\text {th }}$ iteration, and

- Antlion $_{j}^{t}$ is the position of the selected $j^{\text {th }}$ antlion at $t^{\text {th }}$ iteration.

\subsubsection{Sliding ant toward trap}

Ones the ant is in the pit, the antlion stars shooting sands outwards the center of that pit. The behavior of sliding ant toward antlions' traps is formulated using the following equations (Hatata et al. 2018; Mirjalili 2015):

$c^{t}=\frac{c^{t}}{I}$

$d^{t}=\frac{d^{t}}{I}$

$I=10^{w} \frac{t}{T}$

where:

- $t$ is the current iteration,

- $T$ is the maximum number of iterations.

To adjust the accuracy level of exploitation, the constant $w$ is used.

where:

- $\quad w=2$ when $t>0.1 T, w=3$ when $t>0.5 T$ etc...,

\subsubsection{Catch the preys and re-build the traps}

The behavior modelling of catching ant mechanism and rebuilding the traps, is expressed using the following formula:

Antlion $_{j}^{t}=$ Ant $_{i}^{t}$, if $f\left(\right.$ Ant $\left._{i}^{t}\right)>f\left(\right.$ Antlion $\left._{j}^{t}\right)$

where:

- Antlion $_{j}^{t}$ is the position of selected $j^{\text {th }}$ antlion at $t^{\text {th }}$ iteration, and

- $A n t_{i}^{t}$ is the position of $i^{t h}$ ant at $t^{t h}$ iteration.

\subsubsection{Elitism}

In each iteration, the best antlion obtained is considered as elite (Mirjalili 2015). It is maintained as the fittest solution. Therefore, the random walks of ants are assumed to be affected by their movements around a selected antlion based on the roulette wheel operator and the elite simultaneously as follows:

$A n t_{i}^{t}=\frac{R_{A}^{t}+R_{E}^{t}}{2}$

where:

- where $R_{A}^{t}$ is the random walk around the antlion selected by roulette wheel at $t^{\text {th }}$ iteration,

- where $R_{E}^{t}$ is the random walk around the elite at $t^{\text {th }}$ iteration.

\section{Results and Discussions}

This section investigates the implementation and simulation of the developed EMS to the MG under study. The EMS was evaluated with a time step of $\Delta \mathrm{T}=15 \mathrm{~min}$ during $24 \mathrm{~h}$ time interval. Modelling and simulation of the optimization problem were conducted using MATLAB/Simulink environment. The purpose of the developed system is to ensure power management optimization with high level penetration of RESs, realtime operating, emission and power loss costs functions minimization, taking into account the previous constraints mentioned earlier. The maximum supply capacity of DG, FC, and MT is set to $40 \mathrm{kw}, 50 \mathrm{kw}$ and 65 
$\mathrm{kw}$, respectively. The maximum exchanged power with the main grid is limited to $50 \mathrm{kw}$. Finally, the maximum charging and discharging power of ESS is fixed to $20 \mathrm{kw}$, while its SoC is bounded between $20 \%$ and $80 \%$. The validity and effectiveness of the proposed approach are evaluated under both grid connected and standalone mode. Then, a comparison results with other well-known optimization algorithms include basic PSO, ABC and NBA approaches is detailed. The obtained simulation results are described hereafter.

\subsection{Policy 1: Microgrid running under grid connected mode}

In this scenario, the battery is initially charged to $50 \%$ of SoC. The ESS participates beside the two RESs to cover the power demand. Fig. 6 shows the corresponding output power from the RESs and the SoC evolution. It can be observed that, the battery's SoC is maintained at optimum levels, avoiding full charges-discharges cycles. The optimal charging and discharging powers of ESS is shown in Fig. 7. During the simulation time, the available power from the two RESs in MPPT mode and the battery power supply during discharging cycles are not enough to cater the required demand. Therefore, the EMS decides to start up the CESs, while start import power from the main grid. Fig. 8 shows the optimal output power of each CES and the imported power from main grid, respectively. It can be seen that, under grid connected mode, the FC unit and the main grid are prior scheduled since they propose lower costs. The DG unit though, was dispatched less time with fewer generated power. The contribution rate of each power generation source of the studied MG is shown in Fig. 9. It is clearly seen, that the total load demand is supplied by $52.26 \%$ CESs, contrary to RESs and ESS's discharging cycles, which their contribution is limited to $17.74 \%$. Finally, Fig. 10 reveals the total system power balance for a day ahead time horizon and, Fig. 11 describes a real time MG's overall cost under operation policy 1.

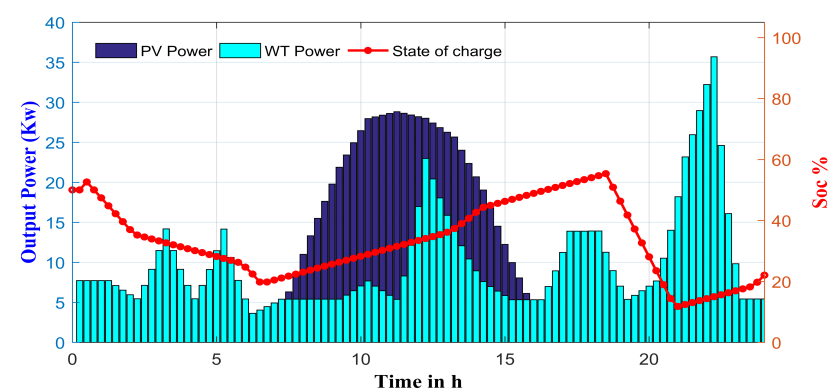

Fig. 6 Output power of RESs in grid connected mode under scheduling policy 1

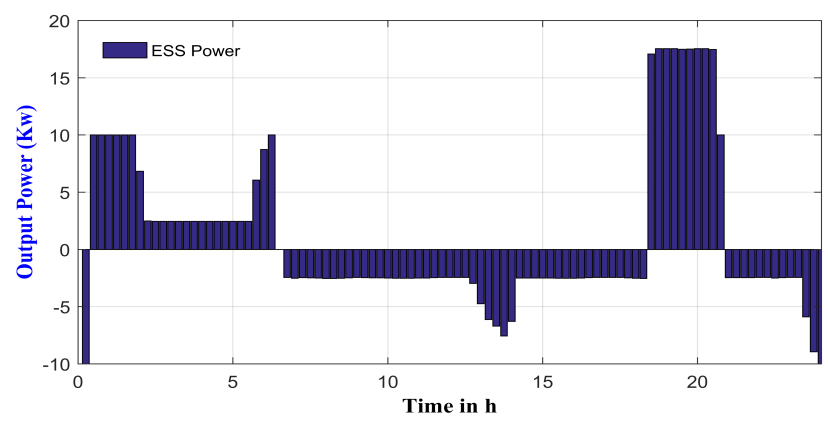

Fig. 7 Optimal charging/discharging of battery storage system under scheduling policy 1

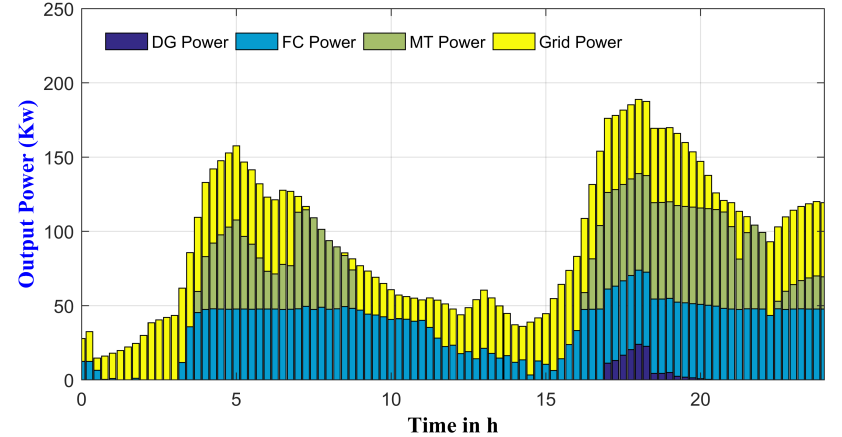

Fig. 8 Optimal power allocation of CESs and grid under scheduling policy 1

Policy 1: Under Grid connected Mode

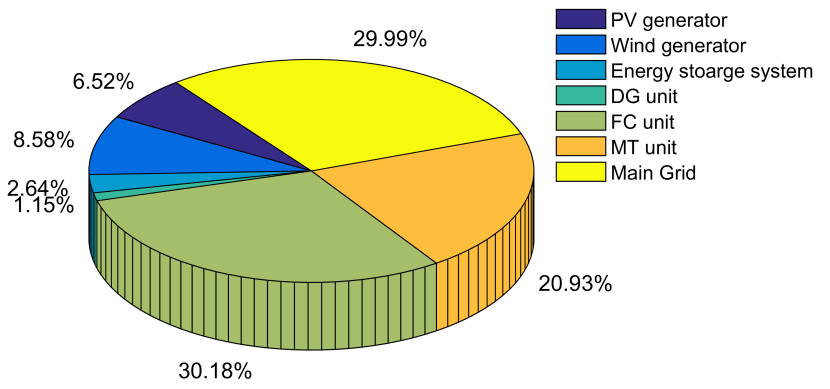

Fig. 9 Contribution of HMG's components in power management under scheduling policy 1

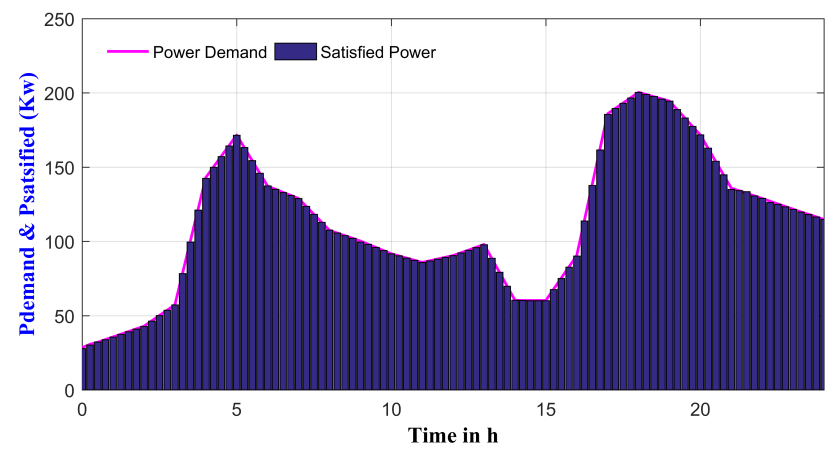

Fig. 10 Total system power balance under scheduling policy 1

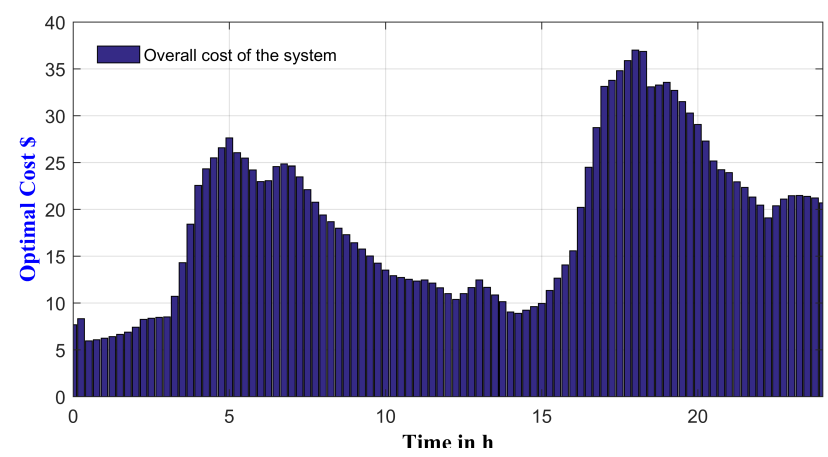

Fig. 11 Overall system's cost under scheduling policy 1

\subsection{Policy 2: Microgrid running under standalone mode}

In this policy, the MG is running under the standalone mode. Due to the strategy to maximize the penetration of RESs, they are used first. However, the load demand still exceeds the combined output power of the RESs and the battery supply limitation, therefore, the CESs subscribe in the scheduling strategy. 
Table 4

Detail of daily simulation results under both operation policies

\begin{tabular}{lcc}
\hline Output Variables & Policy 1 & Policy 2 \\
\hline Daily Overall Cost (\$/day) & 1814.9706 & 1904.5788 \\
Daily Power Generated by RESs (kwh) & 1677.8502 & 1677.8502 \\
Daily Power Generated by CESs (kwh) & 5770.5952 & 8909.5404 \\
Daily Exchanged Power with the grid (kwh) & 3366.0362 & 0 \\
Daily Demand Reduction (kwh) & 5.0120 & 227.5611 \\
Daily Power Loss of conversion (kwh) & 226.0731 & 221.5313 \\
Daily Emission level of $\mathrm{NO}_{\mathbf{x}}(\mathrm{kg} / \mathrm{day})$ & 7.1608 & 9.7277 \\
Daily Emission level of $\mathrm{SO}_{2}(\mathrm{~kg} /$ day) & 6.1079 & 0.2112 \\
Daily Emission level of $\mathrm{CO}_{2}(\mathrm{~kg} /$ day) & 6.3748 & 5.3450 \\
\hline
\end{tabular}

Fig. 12 shows the optimal output power of dispatchable sources. It can be seen, that whenever ESS is in the discharging mode (supplying power) the participation of the CESs decreased. However, when ESS is in charging mode (demanding power) the participation of the CESs became more important to cover the load and charge the battery. Moreover, it is observed from the results that the DG is the least desirable production option since it provides the highest costs.

During 16h45min-19h30min the load demand exceeds each of RESs and CESs supply limitation, so to ensure steady operating system and satisfy the inequality constraints of the MG's components, the FLS switches the load to the constrained mode where all DERs (RESs, CESs, and battery in case of discharging) meet the power demand of the critical load. As shown in Fig. 13, a load shedding option is introduced, turning a part of the controllable demand off while keeping the supply of the critical one. Finally, Fig. 14, show the contribution rate of each element of the studied system, under standalone mode. While, Table 4 summarizes the data obtained under both operation policies. From an economic point of view, it is obvious that, running the system under grid connected mode is more beneficial, since the overall cost is reduced by $4.70 \%$. However, the pollutant gas emissions under policy 2 is lower compared when the system is running under grid connected mode.

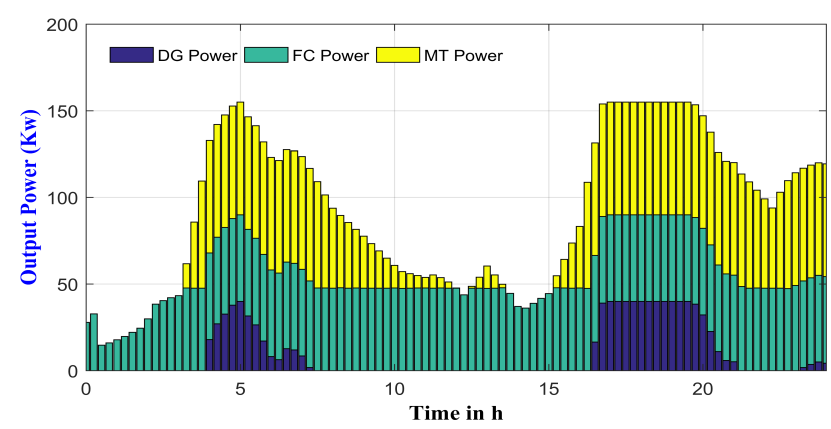

Fig. 12 Optimal power allocation of CESs under scheduling policy 2

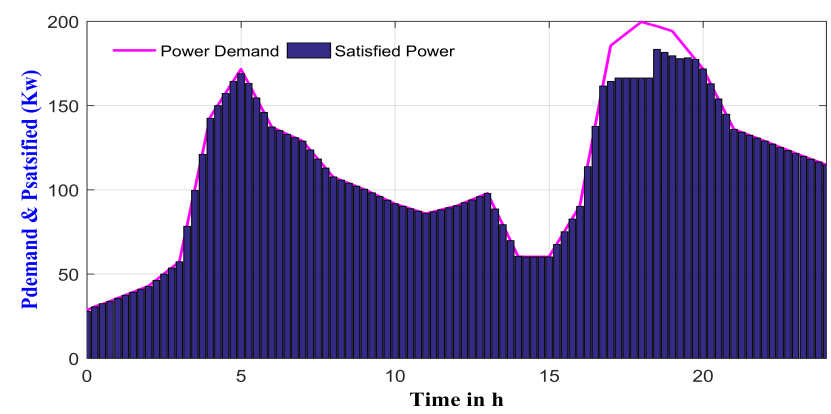

Policy 2: Under Standalone Mode

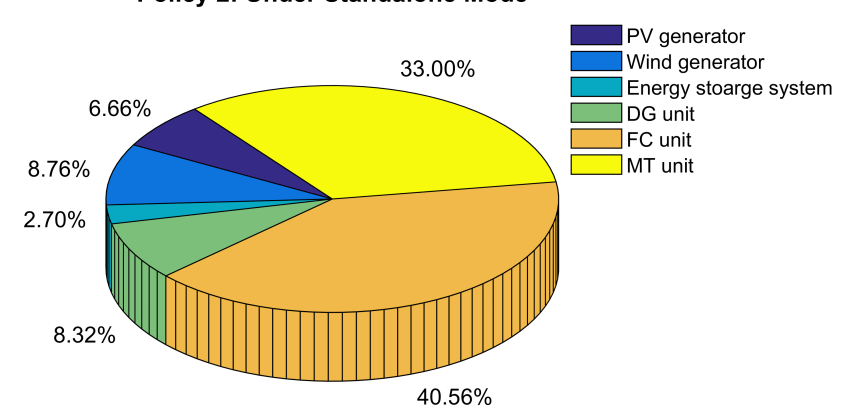

Fig. 14 Contribution of HMG's components in power management under scheduling policy 2

\subsection{Comparison results}

For comparison purpose, the multi-objective optimization dispatch problem was minimized using ALO and the other heuristic algorithms. Table 5 shows the performance comparison based on a fixed load demand $P_{D}=72.125 \mathrm{kw}$, under both operation policies, calculated for 100 trial runs.

It is clear that the proposed ALO approach is more cost effective, it could find better optimal solutions compared to other proposed algorithms. Also, it is to be noted, from the obtained best cost (BC), worst cost (WC), mean cost (MC) and standard deviation (SD) values, that the proposed method has the best performance and provides better robustness and stability than basic PSO and the other heuristic algorithms. Fig. 15 illustrates the comparison of the convergence curves of the best function values under both operation policies based on a fixed load demand. Compared to the other heuristic algorithms, it is shown that, the ALO approach converges better to the global optimal solution.

Table 5

Performance comparison under both operating policies based on a fixed load demand calculated using ALO and the other heuristic algorithms for 100 trial runs

\begin{tabular}{|c|c|c|c|c|c|}
\hline & & Basic PSO & $\begin{array}{c}\text { NBA } \\
\text { algorithm }\end{array}$ & $\begin{array}{c}\mathrm{ABC} \\
\text { algorithm }\end{array}$ & $\begin{array}{c}\text { ALO } \\
\text { algorithm }\end{array}$ \\
\hline \multirow{4}{*}{$\begin{array}{l}\text { - } \\
\stackrel{0}{0} \\
0 \\
0\end{array}$} & $\mathrm{BC}$ & 13.584157 & 13.584157 & 13.584774 & 13.584156 \\
\hline & WC & 13.586313 & 16.589862 & 14.440799 & 13.584157 \\
\hline & $\mathrm{MC}$ & 13.584802 & 14.384072 & 13.926871 & 13.584157 \\
\hline & $\mathrm{SD}$ & $5.299459 \mathrm{e}-04$ & 1.205031 & 0.208777 & $3.749407 \mathrm{e}-08$ \\
\hline \multirow{4}{*}{ 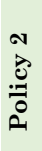 } & $\mathrm{BC}$ & 15.654853 & 15.654805 & 15.654810 & 15.654804 \\
\hline & WC & 15.658740 & 16.582264 & 15.724748 & 15.654819 \\
\hline & MC & 15.656024 & 15.879509 & 15.657859 & 15.654806 \\
\hline & $\mathrm{SD}$ & $8.548499 \mathrm{e}-06$ & 1.205031 & 0.007329 & $2.578965 \mathrm{e}-06$ \\
\hline
\end{tabular}

Fig. 13 Total system power balance under scheduling policy 2 


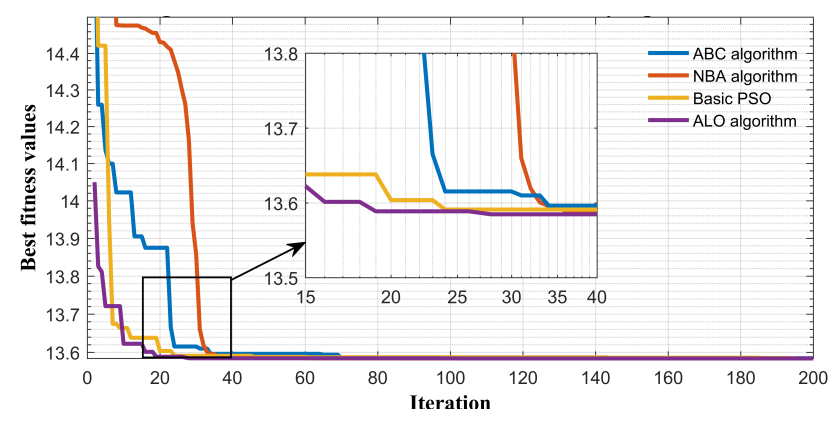

(a)

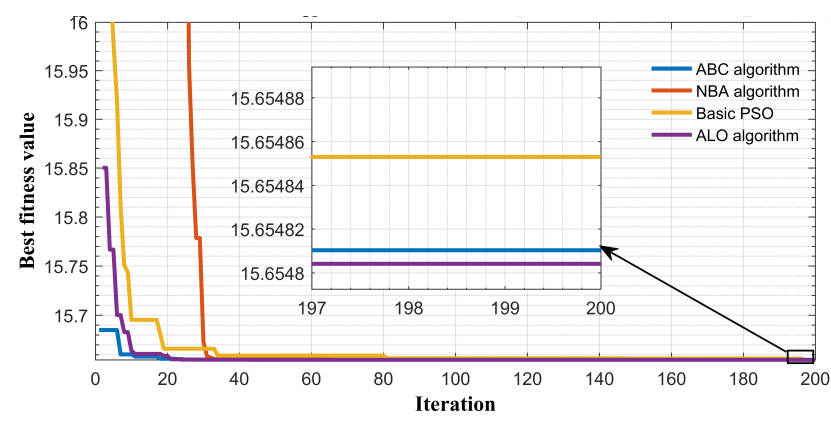

(b)

Fig. 15 Comparison of the convergence curves of ALO and other heuristic algorithms under (a) grid connected mode, (b) standalone mode.

Also the proposed algorithm provides good performance in terms of convergence speed and time, since it requires less time and fewer iterations to converge to the global optimal solution. It is also to be mentioned, that in each optimization algorithm, the number of population is set to $N_{p}=40$, and the maximum number of iterations is limited to 200 .

In summary, we think that introducing ALO algorithm to solve the overall objective function, leads to considerable reduction in the total cost, and confirm the capability and efficiency of the approach to deal better with the MOOD problem of the studied MG.

\section{Conclusion}

In this paper, we presented a novel energy management system for day-ahead optimal power management and control of a MG, composed of various types of distributed generators such as photovoltaic arrays, wind turbine, diesel generator, micro turbine, fuel cell and battery storage system.

The proposed EMS is designed to operate in two layers' structure, combining fuzzy logic system as the main controller with ant lion optimizer algorithm dedicated to solve the multi-objective optimization dispatch problem.

Three conflicting objectives functions are considered for minimization: the operating cost, emission of the pollutants levels and power loss cost of conversion devices. Taking into account optimal scheduling of battery's charging/discharging cycles, control of load curtailment and the system technical constraints. To evaluate the performance of the developed model, two different operation policies grid connected and standalone mode are investigated, and a comparison with other heuristic methods is proposed. The obtained simulation results demonstrate the effectiveness and consistency of the proposed algorithm to achieve global optimal solution and in fewer iterations. It can be summarized that the proposed system is appropriate to solve technical, economic and environmental problems. Consequently, it can be applied for handling the MOOD problem in MG system.

\section{References}

Aghajani, G., \& Ghadimi, N. (2018). Multi-objective energy management in a micro-grid. Energy Reports, 4, 218-225; doi.org/10.1016/j.egyr.2017.10.002.

Al-Sakkaf, S., Kassas, M., Khalid, M., \& Abido, M. A. (2019). An Energy management system for residential autonomous dc microgrid using optimized fuzzy logic controller considering economic dispatch. Energies, 12, 1-25. https://doi.org/10.3390/en12081457

Alazemi, F. Z., \& Hatata A. Y. (2019). Ant lion optimizer for optimum economic dispatch considering demand response as a visual power plant. Electric Power Components and Systems; doi.org/10.1080/15325008.2019.1602799.

Alvarado-Barrios, L., Rodríguez del Nozal, A., Tapia, A., Martínez-Ramos, J. L., \& Reina, D. (2019). An evolutionary computational approach for the problem of unit commitment and economic dispatch in microgrids under several operation modes. Energies, 12, 2143; doi.org/10.3390/en12112143.

Augusto, O., Bennis, F., \& Caro, S. (2012). A New method for decision making in multi-objective optimization problems. Sociedade Brasileira de Pesquisa Operacional, 32 (2), 331-369.

Contreras, S. F., Cortes, C. A, \& Myrzik, J. M. A. (2019). Optimal microgrid planning for enhancing ancillary service provision. Journal of Modern Power Systems and Clean Energy, 7, 862-875; doi.org/10.1007/s40565-019-0528-3.

García, P., García, C. A., Fernández, L. M., F. Llorens, \& Jurado, F. (2014). ANFIS-based control of a grid-connected hybrid system integrating renewable energies, hydrogen and batteries. IEEE Transactions on Industrial Informatics, 10 (2), 1107-1117; doi.org/10.1109/TII.2013.2290069.

Garcia, P., Torreglosa, J. P., Fernandez, L. M., \& Jurado, Fr. (2013). Optimal energy management system for standalone wind turbine/photovoltaic/hydrogen/battery hybrid system with supervisory control based on fuzzy logic. International Journal of Hydrogen Energy, 38 (33), 14146-14158; doi.org/10.1016/j.ijhydene.2013.08.106.

Hatata, A. Y., \& Hafez, A. A. (2019). Ant lion optimizer versus particle swarm and artificial immune system for economical and eco-friendly power system operation. International Transaction on Electrical Energy Systems; doi.org/10.1002/etep.2803.

Jiang, Q., Xue, M., \& Geng, G. (2013). Energy management of microgrid in grid-connected and stand-alone modes. IEEE Transactions On Power Systems, 28 (3), 3380-3389; doi.org/0.1109/TPWRS.2013.2244104.

Jin, X., Mu, Y., Jia, H., Wu, J., Jiang, T., \& Yu, X. (2017). Dynamic economic dispatch of a hybrid energy microgrid considering building based virtual energy storage system. Applied Energy, 194, 386-398; doi.org/10.1016/j.apenergy.2016.07.080.

Kamboj, V. K., Bhadoria, A., \& Bath, S. K. (2017). Solution of nonconvex economic load dispatch problem for small-scale power systems using ant lion optimizer. Neural Computing and Applications, 28, 2181-2192; doi.org/10.1007/s00521-0152148-9.

Karaboga, D., \& Akay, B. (2009). A Comparative study of artificial bee colony algorithm. Applied Mathematics and Computation, 214 (1), 108-132; doi.org/10.1016/j.amc.2009.03.090. 
Kiptoo, M. K., Lotfy, M. E., Adewuyi, O. B., conteh, A., howlader, A. M., \& Senjyu, T. (2020). Integrated approach for optimal techno-economic planning for high renewable energy-based isolated microgrid considering cost of energy storage and demand response strategies. Energy Conversion and Management, 215; doi.org/10.1016/j.enconman.2020.112917.

Kyriakarakos, G., Dounis, A. I., Arvanitis, K. G., \& Papadakis G., (2012). A fuzzy logic energy management system for polygeneration microgrids. Renewable Energy, 41, 315-327; doi.org/10.1016/j.renene.2011.11.019.

Lagouir, M., Badri, A., Sayouti, Y. (2019). Development of an intelligent energy management system with economic dispatch of a standalone microgrid. Journal of Electrical Systems, 15 (4) 568-581.

Liu, H., Ji, Y., Zhuang, H., \& Wu, H. (2015). Multi-Objective dynamic economic dispatch of microgrid systems including vehicle-to-grid. Energies, 8, 4476-4495. DOI: 10.3390/en8054476

Meng, X. B., Gao, X. Z., Liu, Y., \& Zhang, H. (2015). A novel bat algorithm with habitat selection and doppler effect in echoes for optimization. Expert Systems with Applications, 42 (1718), 6350-6364; doi.org/10.1016/j.eswa.2015.04.026.

Mirjalili, S. (2015). The Ant Lion Optimizer. Advances in Engineering Software, 83, 80-98; doi.org/10.1016/j.advengsoft.2015.01.010.

Mohamed, F. A., \& Koivo, H. N. (2012). Online management genetic algorithms of microgrid for residential application. Energy Conversion and Management, 64, 562-568; doi.org/10.1016/j.enconman.2012.06.010.

Moradi, H., Esfahanian, M., Abtahi, A., \& Zilouchian, A. (2018). Optimization and energy management of a standalone hybrid microgrid in the presence of battery storage system. Energy, 147, 226-238; doi.org/10.1016/j.energy.2018.01.016.

Murty, V. V. S. N., \& Kumar, A. (2020). Multi-objective energy management in microgrids with hybrid energy sources and battery energy storage systems. Protection and Control of Modern Power Systems, 5 (2); doi.org/10.1186/s41601-019$\underline{0147-\mathrm{z} .}$

Nemati, M., Braun, M., \& Tenbohlen, S. (2018). Optimization of unit commitment and economic dispatch in microgrids based on genetic algorithm and mixed integer linear programming. Applied Energy, 210, 944-963; doi.org/10.1016/j.apenergy.2017.07.007.

Nwulu, N. I., \& Xia, Xi. (2015). Multi-objective dynamic economic emission dispatch of electric power generation integrated with game theory based demand response programs. Energy Conversion and Management, 89, 963-974; doi.org/10.1016/j.enconman.2014.11.001.

Olivares, D. E., Cañizares, C. A., \& Kazerani, M., (2011). A Centralized optimal energy management system for microgrids. IEEE Power and Energy Society General Meeting; doi.org/10.1109/PES.2011.6039527.

Parisio, A., Rikos, E., \& Glielmo, L., (2014). A Model predictive control approach to microgrid operation optimization. IEEE
Transactions on Control Systems Technology, 22 (5), 18131827; doi.org/10.1109/TCST.2013.2295737.

Reddy, S. S. (2017). Optimal power flow with renewable energy resources including storage. Electrical Engineering, 99, 685695; doi.org/10.1007/s00202-016-0402-5.

Reddy, S. S., \& Momoh, J. A. (2015). Realistic and transparent optimum scheduling strategy for hybrid power system. IEEE Transactions on Smart Grid, 6 (6), 3114-3125; doi: 10.1109/TSG.2015.2406879.

Shen, J., Jiang, Ch., Liu, Y., \& Wang, X. (2016). A Microgrid energy management system and risk management under an electricity market environment. IEEE Access, 4, 2349-2356.

Taha, M. S., Abdeltawab, H., Ha., \& Mohamed, Y. A. I. (2018). An Online energy management system for a grid-connected hybrid energy source. IEEE Journal of Emerging and Selected Topics in Power Electronics, $6 \quad$ (4), 2015 -2030; doi.org/10.1109/JESTPE.2018.2828803.

Triantaphyllou, E., \& Mann, S. H. (1995). Using the analytic hierarchy process for decision making in engineering applications: some challenges. International Journal of Industrial Engineering: Applications and Practice, 2 (1), 3544.

Vivas, F. J., Segura, F., Andújar, J. M., Palacio, A., Saenz, J. L., Isorna, F., \& López, E. (2020). Multi-objective fuzzy logicbased energy management system for microgrids with battery and hydrogen energy storage system. Electronics, 9 (7), 1074; doi.org/10.3390/electronics9071074.

Wang, T., He, X., \& Deng, T. (2017). Neural Networks for power management optimal strategy in hybrid microgrid. Neural Computer \& Application Journal, 31 (7), 2635-2647.

Wang. Z., Zhu, Q., Huang, M., \& Yang, B. (2017). Optimization of economic/environmental operation management for microgrids by using hybrid fireworks algorithm. International Transactions on Electrical Energy Systems, 27 (12); doi.org/10.1002/etep.2429.

Wu, H., Liu, X., \& Ding, M. (2014). Dynamic economic dispatch of a microgrid: Mathematical models and solution algorithm. Electrical Power and Energy Systems, 63, 336-346; doi.org/10.1016/j.ijepes.2014.06.002.

Wu, H., Zhuang, H., Zhang, W., \& Ding, M. (2016). Optimal allocation of microgrid considering economic dispatch based on hybrid weighted bilevel planning method and algorithm improvement. Electrical Power and Energy Systems, 75, 2837; doi.org/10.1016/j.ijepes.2015.08.011.

Wu, X., Cao, W., Wang, D., \& Ding, M. (2019). Multi-objective optimization dispatch method for microgrid energy management considering the power loss of converters, Energies.12(11), 2160, https://doi.org/10.3390/en12112160

Yuan, X., Zhang, B., Wang, P., Liang, J., Yuan, Y., Huang, Y., \& Lei, X. (2017). Multi-objective optimal power flow based on improved strength Pareto evolutionary algorithm. Energy, 122, 70-82; doi.org/10.1016/j.energy.2017.01.071. 